\title{
Zur Verfassungsentwicklung in Marokko
}

\author{
Von Silvia Tellenbach
}

Am 4. September 1992 wurden in einem Referendum umfangreiche Änderungen der marokkanischen Verfassung beschlossen und diese bald darauf neu verkündet ${ }^{1}$. Seit dem Inkrafttreten der ersten Verfassung von 1962, 6 Jahre nach Erlangung der Unabhängigkeit, war dies bereits die vierte Neuverkündung ${ }^{2}$. Wie sich auch bei anderen Ländern der Region erkennen läßt ${ }^{3}$ gelten Verfassungen nicht in dem uns gewohnten Maße als besonders bestandsfeste Gesetze, sondern werden rascher geändert oder gar ersetzt. Politische Veränderungen, ja schon sich wandelnde Tendenzen, schlagen sich leichter auch in den Verfassungen nieder ${ }^{4}$.

Um die im folgenden zu behandelnden Änderungen in einen Rahmen stellen zu können, sollen hier zunächst einige Grundstrukturen der bisherigen Verfassung skizziert wer$\operatorname{den}^{5}$.- Die marokkanische Verfassung lehnt sich in ihren äußeren Formen, in Aufbau und Regelungsmaterie an die uns aus Europa vertrauten Verfassungen an, wobei das Vorbild der französischen Verfassung von 1958 stellenweise sehr deutlich sichtbar wird ${ }^{6}$. Auf die Präambel und Grundprinzipien.wie Monarchie, Volkssouveränität, Staatsreligion und einen Katalog der Bürgerrechte folgt die Staatsorganisation mit dem König als Staatsoberhaupt, Regierung, Parlament und Justiz, schließlich eine Ewigkeitsgarantie für die

1 Bulletin Officiel no. 4173 v. 21.10 .1992

2 Zur Vorgeschichte vgl. H. Mattes, Moderne arabische Verfassungsentwicklung, in: Demokratie und Menschenrechte, S. Faath/H. Mattes (Hrsg.), Hamburg 1992, 49 ff. (59 ff.); S. Faath, Marokko. Die innen- und außenpolitische Entwicklung seit der Unabhängigkeit. Kommentar und Dokumentation. 2 Bände, Hamburg 1987; ein kurzer Abriß der Verfassungsgeschichte findet sich bei $D$. Basri, L' évolution constitutionnelle au Maroc depuis 1962, in: Trente années de vie constitutionnelle au Maroc, D. Basri/M. Rousset/G. Vedel (Hrsg.), Paris 1993, 75 ff.

z.B. Algerien, Syrien.

4 Mattes, a.a.O., 50.

5 Text der marokkanischen Verfassung von 1972 und Einführung bei $C$. Amelunxen, Staatsaufbau und Verfassungsentwicklung in Marokko (1908-1988). Jahrbuch für öffentliches Recht 38 (1989), $499 \mathrm{ff}$; eine kurze Einführung auch bei $S$. Faath, Rechte und Freiheiten der Staatsbürger im "Hassanismus", in: Demokratie und Menschenrechte, S.Faath/H.Mattes (Hrsg.), Hamburg 1992, $365 \mathrm{ff}$. (368 ff.)

6 Ein Vergleich der marokkanischen und der französischen Verfassung bei G. Vedel, Royaume du Maroc et République Française - Parallèle entre deux Constitutions, in: Trente années de vie constitutionnelle au Maroc, D. Basri/M. Rousset/G. Vedel (Hrsg.), Paris 1993, $363 \mathrm{ff}$. 
Monarchie und den Islam als Staatsreligion ${ }^{7}$. Der inneren Substanz nach gibt es aber deutliche Unterschiede. Sie beruhen vor allem auf der starken Stellung des Königs (Art. 19), der als "Fürst der Gläubigen" gleichzeitig auch religiöses Oberhaupt ist ${ }^{8}$ und so gewissermaßen wählen kann, ob er als weltlicher Herrscher den Staatsbürgern oder als religiöses Oberhaupt den Gläubigen gegenübertritt ${ }^{9}$. Der König ist geheiligt und unverletzlich (Art. 23), was schon jegliche Kritik an ihm nahezu verbietet ${ }^{10}$. Die Verfassung sieht für ihn bestimmte staatsrechtliche Funktionen vor, von denen hier nur die wichtigsten zu nennen sind. Viele ähneln denen des französischen Präsidenten, an bedeutsamen Stellen gehen sie jedoch darüber hinaus. Der König wacht über die Achtung der Verfassung (Art. 19) ${ }^{11}$, was auch ihre Interpretation beinhaltet ${ }^{12}$ Der König ernennt und entläßt den Ministerpräsidenten und die Minister (Art. 24), letzteres bisher ohne entsprechenden Vorschlag des Ministerpräsidenten ${ }^{13}$ und führt den Vorsitz im Ministerrat (Art. 25) ${ }^{14}$, er verkündet die vom Parlament verabschiedeten Gesetze (Art. 26) ${ }^{15}$. Der König kann bei jedem Gesetzesentwurf über eine Botschaft an das Parlament eine weitere Lesung verlangen (Art. 66/67) ${ }^{16}$. Entscheidet das Parlament auch jetzt nicht in seinem Sinne, so kann er über das Gesetz direkt in einem Referendum entscheiden lassen, es sei denn, das Parlament habe vorher mit einer Zweidrittelmehrheit über den fraglichen Entwurf

7 Auch die Indemnität der Abgeordneten endet hier, Art. 37 I. Äußerungen gegen die Monarchie, gegen den Islam als Staatsreligion oder Äußerungen, die den Respekt gegenüber dem König vermissen lassen, sind nicht geschützt.

8 Zur Stellung des Königs vgl. M. Torrelli, Le pouvoir royal dans la Constitution, in: Trente années de vie constitutionnelle au Maroc, D. Basri/M. Rosset/G. Vedel (Hrsg.), Paris 1993, 107 ff.; M Tozy, Le Roi Commandeur des croyants, in: Édification d'un État moderne. Le Maroc de Hassan II, G. Vedel (Hrsg.), Paris 1986, 51 ff.; S. Faath, " Le Hassanisme". Das marokkanische Konzept von Demokratie, Wuquf 4-5, Hamburg 1991, 9 ff.; S. Faath 1992, 367;, vgl auch A. Menouni, Séparation des pouvoirs et Constitution, in: Trente années de vie constitutionnelle au Maroc, D. Basri/M. Rousset/G. Vedel (Hrsg.), Paris 1993, 175 ff. (185).

9 Die Frage, wieweit die im islamischen Staat nicht vorhandene Trennung zwischen beiden Bereichen in Marokko noch fortwirkt, ist hier nicht zu behandeln.

10 M.A. Bakhechi, Lecture comparée de la Constitution marocaine et de la Constitution algérienne, in: Trente années de vie constitutionnelle au Maroc, D. Basri/M. Rousset/G. Vedel (Hrsg.), Paris 1993, 335 ff. (345); selbstverständlich sind auch gerichtliche Schritte gegen Akte des Königs nicht möglich, Bakhechi, a.a.O., m.w.N.

11 Art. 5 I S.1 frz Verf.v. 1958

12 Menouni 1993, 195

13 So aber Art. 8 II frz.Verf., ferner erfolgt die Entlassung des Ministerpräsidenten erst nach Rücktritt der Regierung, Art. 8 I frz.Verf.

14 Vgl. Art. 9 frz.Verf.

15 Vgl. Art. 10 I frz.Verf.

16 Vgl. Art. 10 II frz.Verf., allerdings nicht durch Botschaft des Präsidenten, sondern durch einfache Rückgabe an das Parlament. 
entschieden (Art. 68) ${ }^{17}$.- Unabhängig davon kann der König bei einer von ihm initiierten Verfassungsänderung über diese sogar ohne vorherige Befassung des Parlaments ein Referendum anberaumen (Art. 97 II) ${ }^{18}$. Und der Abstimmungsempfehlung, die der König nicht versäumt, seinem Volk zu erteilen, werden sich nur wenige entziehen. Überdies kann der König das Parlament auflösen (Art. 27) und hat ferner weitreichende Befugnisse, auch zum Normenerlaß, wenn zwischen zwei Wahlen kein Parlament existiert (Art. 71 II). Außerdem ist er Oberbefehlshaber der Streitkräfte und hat das Monopol in der Außenpolitik, Art. $31^{19}$. Schließlich hat der König das Recht, den Notstand auszurufen, (Art. 35) zwar nur, wenn bestimmte Notsituationen eingetreten sind und nach Konsultationen mit dem Präsidenten des Parlaments, die zusätzlichen Sicherungen der französischen Regelung (Art. 16 frz. Verf.) fehlen jedoch, die für die Ausrufung des Notstands eine "schwere und unmittelbare" Gefahr, das Unterbrechen der normalen staatlichen Funktionen und bei den Notstandsmaßnahmen den Willen zu einer unverzüglichen Wiederherstellung des regulären Funktionierens der Staatsorgane verlangen ${ }^{20}$. Zwar hat der Ministerpräsident grundsätzlich ein Gegenzeichnungsrecht, dieses entfällt jedoch gerade bei besonders wichtigen Handlungen des Königs ${ }^{21}$ wie z.B. Ausrufung des Notstands, Anberaumung eines Referendums, wenn das Parlament nicht in seinem Sinne abgestimmt hat oder für eine Verfassungsänderung, Auflösung des Parlaments und Besetzung wichtiger Positionen, etwa die des Ministerpräsidenten (Art. 29). In den Händen des Königs liegt also die entscheidende $\mathrm{Macht}^{22}$.

17 Der französische Präsident hat diese Möglichkeit nicht. Er kann ein Referendum über einen Gesetzesentwurf nur auf Antrag der Regierung oder der beiden Kammern anberaumen und das auch nur bei bestimmten Gesetzesentwürfen (Art. 11 frz. Verfassung). Das Verlangen des Präsidenten nach einer weiteren Lesung dient, abgesehen won der Korrektur technischer Fehler, in Frankreich dazu, die Verfassungswidrigkeit eines Gesetzes geltend zu machen. Der Präsident hat bisher von dieser Möglichkeit, die sich einem Vetorecht annähert, äußerst selten Gebrauch gemacht, J. Gicquel, Droit constitutionnel et institutions politiques, $10^{\mathrm{e}}$ éd., Paris 1989, $680 \mathrm{f}$.

18 Während ein Parlamentsentwurf für eine Verfassungsänderung zunächst eine Zweidrittelmehrheit finden muß, bevor er dem Volk zur Abstimmung vorgelegt wird (Art. 98).

19 A. Hasbi/S. Ihrai, Constitutions marocaines et environnement international, in: Trente années de vie constitutionnelle au Maroc, D. Basri/M. Rousset/G. Vedel (Hrsg.), Paris 1993, 27 ff. (39).

20 Nicht zu verwechseln mit dem Ausnahmezustand gem Art. 48, der nach Befassung des Ministerrats durch (königlichen) Dahir ausgerufen wird und nach 30 Tagen nur durch Gesetz verlängert werden kann. Dieselbe Unterscheidung, wenn auch im Detail mit anderen Regelungen, gibt es im französischen Verfassungsrecht, vgl. Art. 18 und Art. 36 frz.Verf.

21 Auch beim französischen Präsidenten entfällt in diesen Fällen die Gegenzeichnung, er ist aber stärker an Beratung oder Mitwirkung anderer Institutionen gebunden.

22 Vgl. A. Menouni 1993, 185: pouvant intervenir aux points nodaux du système constitutionnel, il est le seul à pouvoir saisir le peuple par voie de référendum, il peut dissoudre le parlement, demander une seconde lecture, proclamer l'état d'exception, autant de prérogatives qu'il exerce à titre personnel et sans le contreseing du premier ministre. 
Aufgabe der Regierung ist in erster Linie die Ausführung der allgemeinen Politik des Staates, die jedoch letzlich vom König und seinem engsten Beraterkreis festgelegt wird ${ }^{23}$, die Stellungnahme zu Gesetzesentwürfen und der Erlaß von Rechtsnormen (Art. 65).- Die Regierung hängt vor allem vom Vertrauens des Königs ab. Das Parlament kann zwar ein Mißtrauensvotum gegen sie durchbringen (Art. 75), aber in der Praxis ist das nicht leicht. Ein Mißtrauensvotum setzt die Mehrheit der Stimmen aller Parlamentsmitglieder, nicht nur der anwesenden voraus, und eine solche erscheint bei den Mehrheitsverhältnisssen im marokkanischen Parlament ${ }^{24}$ meist wenig aussichtsreich. Gleiches gilt mutatis mutandis auch für die Vertrauensfrage des Ministerpräsidenten.- Auch sonstige Kontrollmöglichkeiten waren gering ausgeprägt ${ }^{25}$. So waren zwar Fragerechte (Art. 55 II), aber keine Untersuchungsausschüsse vorgesehen. Gegenüber dem Parlament hat die Regierung also eine starke Stellung.

Das marokkanische Parlament ist zu zwei Dritteln aus Abgeordneten zusammengesetzt, die direkt gewählt werden (Art. 43). Das dritte Drittel wird indirekt von Vertretern von Gemeinden, berufsständischen Vereinigungen und Arbeitnehmern gewählt, eine Kombination, die aus der Aufgabe des ursprünglich vorgesehenen Zweikammersystems $1970 \mathrm{zu}$ erklären ist. Marokko ist das einzige Land der arabischen Welt, in dessen Verfassung das Einparteiensystem ausdrücklich verboten wird (Art. 3) ${ }^{26}$. Es hat sich auch tatsächlich ein Parteiensystem etabliert, so daß eine relative Meinungsvielfalt zum Ausdruck kommen $\operatorname{kann}^{27}$. Bei Abstimmungen wird jedoch erwartet, sich dem vom König favorisierten Ergebnis anzuschließen ${ }^{28}$.

Zwar ist die Gesetzgebung die wichtigste Aufgabe des Parlaments, es werden aber durchaus nicht alle Rechtsnormen, die in Marokko erlassen werden, vom Parlament erlassen, sondern nur diejenigen, die verfassungsmäßig als Parlamentsangelegenheit festgelegt sind. Inhaltlich sind das vornehmlich der Schutz der Grundrechte und -freiheiten im

23 B.M. Weischer, Das Parteienspektrum und die Rolle des Parlaments in Marokko, KAS-Auslandsinformationen 7/1994, 112 ff. (112).

24 Zum Parlament A. Belhaj, Le Parlement marocain, in: Édification d'un État moderne - le Maroc de Hassan II, G. Vedel (Hrsg.), Paris 1986, 71 ff.; vgl auch $R$. Ben Achour, La révision de la Constitution marocaine de 1992 (4 septembre 1992), Revue française de Droit constitutionnel 15 (1993), 649 ff. (650): parlamentarisme tronqué.

25 F. Zhiri, Réflexions sur l'exercice de la fonction de contrôle de la Chambre des Représentants durant la législature 1977-83, in: L'expérience parlementaire au Maroc, T. Bahbouhi/A. Claisse/A. El Kadiri u.a. (Hrsg.), Casablanca 1985, $95 \mathrm{ff}$.

26 Vgl. zur Begründung des Königs Menouni 1993, 181

27 Vgl. zu den Parteien und ihrer Rolle im Parlament Weischer, a.a.O., $112 \mathrm{ff}$.

28 Faath 1992, 379. 
Zivilrecht, Strafrecht, Verwaltungsrecht und im politischen Bereich (Art. 45) ${ }^{29}$. Andere Rechtsvorschriften werden vom Premierminister erlassen, dem die Befugnis zum Erlaß von Rechtsnormen mit Gesetzescharakter (pouvoir réglementaire) zusteht (Art. 62) ${ }^{30}$. Zur Ratifizierung von völkerrechtlichen Verträgen, die dem König zusteht, ist nur dann eine vorherige Einwilligung des Parlaments erforderlich, wenn diese finanzielle Verpflichtungen mit sich bringen (Art. 31 II) ${ }^{31}$.

Die Opposition, vorweg die Istiqlal-Partei (PI) und die Union Socialiste des Forces Populaires(USFP), hatte schon seit langem Änderungen der Verfassung gefordert, in erster Linie weil ihr die verfassungsmäßigen Möglichkeiten für die Wahrnehmung ihrer Rolle als Opposition zu schwach waren ${ }^{32}$. Es war jedoch schon ein Stachel geblieben, seitdem der König 1972, nachdem er zunächst viele Gespräche auch mit der Opposition geführt hatte, an dieser vorbei auf einmal eine Verfassung zur Volksabstimmung vorgelegt hatte ${ }^{33}$. Seit 1989 hatten mehrere Parteien entsprechende Resolutionen gefaßt und dem König zweimal Memoranden unterbreitet ${ }^{34}$, das erste im Oktober 1991 durch PI und USFP, das zweite im Juni 1992 durch den Demokratischen Block ${ }^{35}$.- Die Notwendigkeit einer Verfassungsänderung, allerdings aus anderen Gründen, hatte sich auch gezeigt, als

29 Was die eingebrachten Entwürfe eingeht, so stammt die weitaus größte Zahl sowieso aus den Ministerien. Das ist jedoch eine internationale Erscheinung, da die Ministerien viel eher über den Apparat verfügen, der zur Ausarbeitung von Gesetzen nötig ist. Zur gesetzgeberischen Tätigkeit des Parlaments im einzelnen vgl. A. Belhaj, L'action du Parlement en matière législative depuis 1977, in: Trente années de vie constitutionnelle au Maroc, D. Basri/M. Rousset/G. Vedel (Hrsg.), Paris 1993, 457 ff.: von 1977-83 wurden 131 Regierungsentwürfe und 14 Parlamentsentwürfe Gesetz, 1984-92 war das Verhältnis noch krasser: 200 Regierungsentwürfe und 13 Parlamentsentwürfe (477). Vgl. auch $M$. Sehimi, Le bilan de la Chambre des Représentants (1977-1983), in: L'expérience parlementaire au Maroc, T. Bahbouhi/A. Claisse/A. El Kadiri u.a. (Hrsg.), Casablanca 1985, 147 ff. (148 ff.); $K$. Naciri, Le Parlement sur le créneau de la production législative quantitative, in: L'expérience parlementaire au Maroc, T. Bahbouhi/A. Claisse/A. El Kadiri u.a. (Hrsg.), Casablanca 1985, $113 \mathrm{ff}$ (126 ff.).- Die Ministerialentwürfe sind vor Einbringung ins Parlament im Ministerrat zu diskutieren, in dem der König wiederum den Vorsitz führt (Art. 25), der so die Entscheidungsinstanz par excellence bleibt.

30 Auch hier findet sich in Frankreich eine ähnliche Aufteilung, nur daß der Katalog der durch Gesetzgebung zu regelnden Materien erheblich umf angreicher ist, Art. 34, 37 frz.Verf.

31 A. Hasbi/S. Ihrai, a.a.O., 40, weisen darauf hin, daß sich jedoch in der marokkanischen Praxis das Parlament zu völkerrechtlichen Verträgen von einschneidender Bedeutung für das nationale Leben habe äußern können.

32 Eingehend zu den Bestrebungen der Oppositionsparteien für eine Verfassungsänderung A. Gallab, Attatawwur ad-dusturi wan-niyabi bil-Magrib (Verfassungsentwicklung-und parlamentarische Entwicklung in Marokko), 3. Aufl., Casablanca 1993, $325 \mathrm{ff}$.

33 O. Bendourou/M. Aouam, La Réforme Constitutionnelle Marocaine de 1992, Revue du Droit Public et de la Science Politique en France et à l'Étranger 109 (1993), 431 ff. (432).

34 Näher dazu Gallab, a.a.O., 332, 336.

35 Dieser wurde im Mai 1992 von fünf Parteien gegründet, außer PI und USFP schlossen sich folgende Gruppierungen an: Parti du Progrès et du Socialisme (PPS), Organisation de l'Action Démocratique et Populaire (OADP) und Union Nationale des Forces Populaires (UNFP). 
in den letzten Jahren die Menschenrechtsdiskussion immer stärker geworden war, und der Zusammenbruch undemokratischer Regime in Osteuropa hatte die Antriebe zur Demokratisierung noch verstärkt ${ }^{36}$.

In der Königsrede vom 3. März 1992 gab der König die Absicht kund, eine Verfassungsänderung einzuleiten, weil politische Institutionen bei all ihren Verdiensten nicht unveränderlich und unberührbar wären und sich mit der weitergehenden Entwicklung auch die politischen Institutionen ändern müßten ${ }^{37}$. Dabei kündigte er an, daß sich die Veränderungen vor allem auf das Verhältnis zwischen Parlament und Regierung beziehen wür$\operatorname{den}^{38}$. Dieser Schritt dürfte maßgeblich von dem Memorandum von PI und USFP vom Herbst 1991 mitbestimmt gewesen sein. Bei der Erstellung des Verfassungsentwurfs wurden als Beteiligte vor allem die Berater des Königs, Ahmed Réda Guédira und Driss Slaoui, der ehemalige Premierminister Ahmed Osman und der Innenminister Driss Basri genannt. Aber auch Anregungen von anderer Seite wurden sichtbar, insbesondere durch die beiden Memoranden vom Herbst 1991 und Sommer 1992, die an vielen Stellen Einfluß ausgeübt zu haben scheinen ${ }^{39}$ Dabei waren Förderung von Rechtsstaatlichkeit und ein neues ausgeglicheneres Kräfteverhältnis zwischen dem Parlament und der Regierung die wichtigsten Nenner, auf die sich die angestrebten Änderungen bringen lassen .

Viel beachtet und viel zitiert ist die Ergänzung der Präambel, gemäß der Marokko seine Bindung an die Menschenrechte erklärt, wie sie international anerkannt sind ${ }^{40}$. Die erste Frage ist jedoch, wieweit die Präambel in sich nur eine Absichtserklärung ist oder wie weit sie geltendes Recht schafft und wenn ja, wie dieses aussähe. Eine eindeutige Formu-

Bendourou/Aouam, a.a.O., 432; Ihrai, Huquq al-insan fi masru ad-dustur al-muraga (Die Menschenrechte im Entwurf einer revidierten Verfassung), in: Muraga at ad-dustur al-magribi 1992 (Die Revision der marokkanischen Verfassung 1992), 62 ff. (65)

37 Discours et Interviews de S.M. Le Roi Hassan II, Hrg. Royaume du Maroc, Ministère de l'Information, Rabat 1993, 15; auch D. Basri, Madhal (Einführung), in: Muraga at ad-dustur al-Magribi (1992) (Revision der marokkanischen Verfassung 1992), D. Basri/M. Rousset/G. Vedel (Hrsg.), o.O. (Rabat) 1992, $11 \mathrm{ff} .(12)$

38 Discours, a.a.O., 15: la révision portera essentiellement sur les dispositions de la Constitution qui organisent les rapports entre le pouvoir executif, l'indépendance du pouvoir judiciaire, choix irréversible, demeurant garantie. Elle s'efforcera d'établir un meilleur équilibre entre les deux pouvoirs, législatif et exécutif, tout en assurant une plus grande justice et une plus grande efficacité dans l'exercice du pouvoir par les responsables qu'ils puisent leur responsabilité d'un mandat populaire ou qu'ils appartiennent à l'administration et au gouvernement, vgl. auch die Rede v. 20.8. Discours, $109 \mathrm{ff}$. (110).

39 Vgl. dazu $M$. Rousset, La révision constitutionnelle, in: Trente années de vie constitutionnelle au Maroc, D. Basri/M. Rousset/G.Vedel (Hrsg.), Paris 1993, $301 \mathrm{ff}$. (316f) und J.M. Montabes/M.A. Parejo, Lecture comparée de la Constitution marocaine et espagnole, in: Trente années de vie constitutionnelle au Maroc, D. Basri/M. Rousset/G. Vedel (Hrsg.), Paris 1993, 393 ff. (410, m.w.N.)

$40 \mathrm{Vgl}$. zu staatlichen und nichtstaatlichen Menschenrechtsorganisationen in den nordaf rikanischen Staaten $H$. Mattes, Menschenrechtsschutz in den arabischen Staaten, in: Demokratie und Menschenrechte in Nordafrika, S. Faath/H. Mattes (Hrsg.), Hamburg 1992, 93 ff. 
lierung für das marokkanische Recht fehlt, wenn wohl auch überwiegend davon ausgegangen wird ${ }^{41}$, daß die Präambel die Bindung an die Menschenrechte zum unmittelbar geltenden Recht macht ${ }^{42}$. Die marokkanische Verfassung enthält auch nicht etwa eine allgemeine Transformationsnorm, durch die bestimmte völkerrechtliche Normen automatisch innerstaatliche Geltung erlangen, vielmehr werden völkerrechtliche Verpflichtungen nur durch Rechtssetzungsakt des Parlaments oder der Exekutive (Art. 45 f.) in innerstaatliches Recht umgesetzt ${ }^{43}$. Im Falle der Menschenrechte ist das jedoch in weitem Umfang geschehen. Marokko hat die beiden Pakte von 1966, den Internationalen Pakt über bürgerliche und politische Rechte und den Internationalen Pakt über wirtschaftliche, soziale und kulturelle Rechte 1979 ratifiziert, und sie sind 1980 in Kraft getreten ${ }^{44}$.- Unabhängig davon ist aber ebenfalls zu bedenken, daß die marokkanische Verfassung einen umfangreichen Katalog von Grundrechten enthält.

Die Ergänzung der Präambel um das Bekenntnis zu den Menschenrechten in ihrer international anerkannten Form bettet sich ein in ein Umfeld, das die Diskussion der Menschenrechte bereits seit einigen Jahren verstärkt aufgenommen hat. Nachdem sich bereits seit einigen Jahren private Menschenrechtsorganisationen etabliert hatten, hat der König 1990 einen Menschenrechtsrat eingesetzt ${ }^{45}$, der auf Zuweisung vom König oder im Wege der Selbstbefassung menschenrechtsrelevante Vorfälle zu untersuchen und Verbesserungsvorschläge zu machen hat. Dieser Rat hat mittlerweile eine Reihe von Gutachten veröffentlicht ${ }^{46}$, denen fast immer gefolgt wurde. Sie haben z.B. dazu geführt, daß im Falle der Festnahme durch die Polizei ("garde-à-vue-Haft") die sofortige Benachrichtigung der Angehörigen zur Pflicht gemacht wurde, weiter ist der Staatsanwaltschaft täglich eine Liste der binnen der letzten 24 Stunden Festgenommenen vorzulegen (Art. 69 StPO), dem Festgenommenen wurde das Recht auf Beiziehung eines Anwalts und auf ärztliche Unter-

41 Ma ida mustadira min tanzim at-talfaza al-magribiya bi-musarakat al-asatida G. Vedel, R.J. Dubois, J. Robert, M. Rousset (Runder Tisch, organisiert vom marokkanischen Fernsehen unter Teilnahme der Professoren G. Vedel, R.J. Dubois, J. Robert und M. Rousset), in: Muraga at ad-dustur al-Magribi 1992 (Die Revision der marokkanischen Verfassung 1992), D. Basri/M. Rousset/G. Vedel (Hrsg.), o.O. (Rabat) 1992, 33 (Dubois); Bendourou/Aouam, a.a.O., 434.

42 Im französischen Recht, von dem das marokkanische Recht stark geprägt ist, gibt es über den juristischen Wert der Präambel drei Auffassungen, einmal, daß die Präambel nur deklaratorischen Wert habe, zum anderen, daß sie geltendes Recht sei, drittens,daß sie Teile habe, die unmittelbar gelten und andere, die der Umsetzung durch den Gesetzgeber bedürften, Bendourou/Aouam, a.a.O., 434.

M.L. Benotmane, Parlement et droits de l'homme, in: Le Parlement et la pratique législative au Maroc, M. Amzazi/A. Ben Messaoud/M. Bennouna u.a. (Hrsg.), Casablanca 1985, $9 \mathrm{ff}$.

Dahir no. 1-79-186 v. 8.11.1979, Bulletin Officiel no. 3525 v. 21.5.1980. 2.5.1990, 306-307, abgedruckt bei Faath/Mattes 1992, 428. Zum Menschenrechtsrat vgl. auch $C h$. Serghini, La Constitution et les libertés publiques, in: Trente années de vie constitutionnelle au Maroc, D. Basri/M. Rousset/G. Vedel (Hrsg.), Paris 1993, $159 \mathrm{ff}$. (170 ff.).

Royaume du Maroc, Conseil Consultatif des Droits de l'Homme o.O., o.J. (Rabat 1993). 
suchung garantiert (Art. 76, $127 \mathrm{StPO})^{47}$ und die Dauer der Untersuchungshaft von regulär vier Monaten mit unbegrenzter Verlängerungsmöglichkeit auf zwei Monate mit Verlängerungsmöglichkeit auf höchstens ein $\mathrm{Jahr}^{48}$ begrenzt (Art. 154 StPO), Punkte, die den beständigen Forderungen von Menschenrechtsorganisationen zur Vermeidung von Folter entsprechen. Zwei weitere wichtige Anliegen, die der Menschenrechtsrat aufgegriffen hat, sind eine umfassende Reform der Strafprozeßordnung und des Strafvollzugsgesetzes und die Stellungnahmen des Rats haben dazu geführt, daß derzeit an einer solchen Reform gearbeitet wird. Noch mehr im Blick der nationalen und internationalen Öffentlichkeit steht das Presserecht, wo jedoch auch in letzter Zeit spürbare Zurückhaltung bei Eingriffen zu bemerken ist ${ }^{49}$. Punkte, an denen derzeit noch Kritik ansetzt, sind z.B. das Vereinigungs- und das Demonstrationsrecht ${ }^{50}$, wo vor allem die Dehnbarkeit von Begriffen wie "Störung der öffentlichen Ordnung" den Bereich eines etwaigen staatlichen Einschreitens sehr ausweiten kann. Energisch wird von der Opposition auch die Abschaffung des Dahir vom 29.Juni 1935 verlangt ${ }^{51}$, dem zufolge mit drei Monaten bis zu zwei Jahren Gefängnis und mit Geldstrafe bestraft werden kann, wer wo und durch welches Mittel auch immer zu Widerstand gegen die Staatsgewalt aufruft, Unruhen oder Umtriebe anstiftet oder provoziert, eine Handlung begeht, die bestrebt ist, die öffentliche Ordnung, Ruhe oder Sicherheit zu stören oder den schuldigen Respekt gegen die Behörden vermissen läßt, Formulierungen, in denen es von unbestimmten Begriffen nur so wimmelt und die dadurch einem menschenrechtswidrigen Mißbrauch Tür und Tor öffnen.

Mit in die Tendenz einer Stärkung der Menschenrechte fällt auch eine kürzlich erfolgte Änderung des Personenstandsgesetzes, das die Stellung der Frau verbessert, die Zwangsverheiratung für sittlich gefährdete Frauen abschafft, das Erfordernis des Heiratsvormunds zumindest für volljährige Frauen, die keinen Vater mehr haben, beseitigt und die Polygamie einschränkt ${ }^{52}$.

Eine auf Anhieb wenig auffällige Vorschrift soll die Geltung von Gesetz und Recht verstärken, nämlich die Vorschrift, daß ein verabschiedetes Gesetz binnen 30 Tagen vom König zu verkünden und damit in Kraft zu setzen ist (Art. 26). Bisher konnte ein Gesetz, das der König zu verkünden nicht für opportun hielt, ad infinitum zurückgehalten wer-

Dahir no. 1-91-110 v. 30.12.1991, Bulletin Officiel no. 4131 v. 1.1.1992.

Höchstens fünfmalige Verlängerung um jeweils zwei Monate.

49 FAZ 6.12.1993.- Hier konnte eine extensive Auslegung des Diffamierungs-und Beleidigungsverbots der Art. 44 ff. Pressegesetz (Dahir 1-58-378 formant Code de la Presse v. 15.11.1958, Bulletin Officiel v. 27.11.1958) problematisch werden, vgl. Faath 1992, 382 ff.

50 Dahir no. 1-58-376 réglementant le droit d'association, Dahir no. 1-58-377 relatif aux rassemblements publics v. 15.11.1958, Bulletin Officiel v. 27.11.1958.

51 Dahir v. 29.6.1935 relatif à la répression des manifestations contraires à l'ordre et des atteintes au respect dû à l'autorité, Bulletin Officiel v. 5.7.1935. Dazu Bendourou/Aouam, a.a.O, 435 f.

Dazu S. Tellenbach, Änderungen im marokkanischen Familienrecht, FamRZ 1994, 943-945. 
den ${ }^{53}$.- Allerdings ist die Formulierung dieser Vorschrift nicht recht geglückt. Es wird nämlich nicht gesagt, was geschieht, wenn die Verkündung dennoch unterbleibt. Soll dann nichts geschehen oder soll das Gesetz dann automatisch in Kraft treten? Hier wäre eine Klarstellung wünschenswert gewesen. ${ }^{54}$

Eine weitere Änderung betrifft die Überprüfung der Verfassungsmäßigkeit eines Gesetzes. Zunächst ist in Erinnerung zu rufen, daß das marokkanische Parlament keineswegs eine Allzuständigkeit für den Erlaß von Gesetzen hat, sondern nur für die in Art. 45 genannten Materien. Auch die Überprüfung von Gesetzen war bisher nur in einem sehr engen Rahmen möglich. Bis jetzt gab es nur eine Kammer beim Obersten Gerichtshof, die nur in engen Grenzen die Verfassungsmäßigkeit einiger weniger Gesetze prüfte (Art. 94 ff. a.F. $)^{55}$. Sie hatte von Amts wegen die Geschäftsordnung des Parlaments und die Organgesetze ${ }^{56}$ zu prüfen und entschied in weiteren bestimmten Fällen auf Vorlage, ob eine Materie zur Regelungskompetenz des Parlaments oder der Exekutive gehörte. In der Praxis gingen Streitigkeiten dabei meist zugunsten der Regierung aus ${ }^{57}$. Die Opposition hatte dann nur noch die Möglichkeit, sich an den König zu wenden und diesen zu einem Eingreifen zu bewegen ${ }^{58}$.

Jetzt wurde nach französischem Vorbild ${ }^{59}$ ein Verfassungsrat eingesetzt (Art. 76 ff.), der mit weiteren Kompetenzen ausgestattet ist. Er ist nicht wie die chambre constitutionnelle per definitionem mit Richtern besetzt, sondern hat 9 Mitglieder, über deren fachliche Qualifikationen keine Aussagen gemacht werden. Eine Befähigung zum Richteramt etwa ist also nicht verfassungsmäßige Voraussetzung für eine Mitgliedschaft. Vier Mitglieder und der Präsident werden vom König ernannt, vier vom Parlamentspräsidenten nach Rücksprache mit den verschiedenen parlamentarischen Gruppen (Art. 77) ${ }^{60}$. Der König

53 Vgl. Rousset, a.a.O., 318, z.B. das Gesetz über die Schaffung einer Verwaltungsgerichtsbarkeit v. 12.7.1991

54 Vgl. auch Bendourou/Aouam, a.a.O., 442.

55 Was fast einheitlich bedauert wurde, $K$. Naciri, La loi et le règlement dans la Constitution, in: Trente années de vie constitutionnelle au Maroc, D. Basri/M. Rousset/G. Vedel (Hrsg.), Paris 1993, $261 \mathrm{ff}$ (295).

56 Verfassungsergänzungsgesetz, grundlegendes Gesetz über die Schaffung neuer oder die Umgestaltung bestehender Staatsorgane.

57 A. Claisse, Le parlement imaginaire, in: L'expérience parlementaire au Maroc, T. Bahbouhi/A. Claisse/A. El Kadiri u.a. (Hrsg.), Casablanca 1985, 56 ff. (61).

58 Naciri, 1993, 298.

59 Vgl. frz. Verf. Art. $56 \mathrm{ff}$.

60 Die Mitglieder wurden im Frühjahr 1994 ernannt, und zwar vom König der Präsident Abbas El Kissi und die Mitglieder Mohamed Naciri, Driss Alaoui Abdellaoui, Abdelaziz Benjelloun und Hassan Kettani (Dahire 1-94-211 bis 1-94-215 vom 28.3.1994) und vom Parlamentspräsidenten die Mitglieder Abderrahman Amalou, Abdeltif Menouni, Mohamed Taquiollah Maadainine, Abdelhadi Benjel- 
hat hier also ein Übergewicht bei der Besetzung. Wieweit dies zum Tragen kommt, hängt allerdings vom Abstimmungsmodus ab (einfache Mehrheit? qualifizierte Mehrheit?) Darüber schweigt die Verfassung. Das mittlerweile ergangene Organgesetz über den Verfassungsrat ${ }^{61}$ sieht jedoch vor, daß Entscheidungen mit Zweidrittelmehrheit zu ergehen haben (Art. 16 III); allein die vom König ernannten Mitglieder können eine Entscheidung also nicht tragen. Ebensowenig äußert sich die Verfassung darüber, ob ein Mitglied eine zweite Amtszeit in dem Rat verbleiben $k^{2} n^{62}$. Auch hier schafft das Organgesetz über den Verfassungsrat inzwischen Klarheit: die sechsjährige Amtszeit kann einmal verlängert werden (Art. 2).

Wie bereits gesagt, kann ein Gesetz nur während des Gesetzgebungsverfahrens überprüft werden $^{63}$. Ist ein Gesetz einmal verabschiedet, so kann seine Verfassungswidrigkeit nicht mehr geltend gemacht werden. Überprüft werden nicht nur Organgesetze und die parlamentarische Geschäftsordnung, vielmehr können alle Gesetze überprüft werden (Art. 79 I, II). Antragsberechtigt für eine Überprüfung sind der König, der Ministerpräsident, der Präsident des Abgeordnetenhauses und ein Viertel der Abgeordneten (Art. 79 III). Das soll auch der Opposition die Möglichkeit sichern, einen Antrag zu stellen. Zur Prüfung hat der Rat 30, in Eilfällen auf Antrag der Regierung nur 8 Tage Zeit (Art. 79 IV). Erklärt er ein Gesetz für verfassungswidrig, so darf es nicht in Kraft treten (Art. 79 VI).- Festgehalten werden sollte in diesem Zusammenhang aber auch, daß der einzelne Bürger, der von einer Verfassungsverletzung betroffen ist, keine gesetzliche Möglichkeit hat, von sich aus den Verfassungsrat anzugehen. Ebenso fehlt etwa die Vorlagemöglichkeit durch ein Gericht. Auf der anderen Seite hat der Verfassungsrat wieder eine Reihe von Prüfungsaufgaben, die einem deutschen Verfassungsgericht abgehen, z.B. bei Wahlen und Referenden (Art. 79 I), ferner wird der Präsident des Verfassungsrats vor der Verhängung des Ausnahmezustands und der Auflösung des Parlaments konsultiert - aber eben nur beratend, nicht bindend für den König.

Eine dritte Gruppe von Änderungen betrifft das Verhältnis von Ministerpräsident und Regierung. Zunächst ist die Stellung des Ministerpräsidenten innerhalb der Regierung an einigen Stellen gestärkt und ihm ausdrücklich die Verantwortlichkeit für die Anwendung der Gesetze übertragen worden (Art. 60).- Während bisher der König den Ministerpräsidenten und die Minister berief und abberief, setzt er jetzt nur den Ministerpräsidenten ein und läßt ihm freie Hand bei der Auswahl der Minister, die er dann auf Vorschlag des

loun (Entscheidungen des Parlamentspräsidenten Nr. 118-94 bis 121-94 vom 21.3.1994), Bulletin Officiel no. 4253 v. 4.51994.

61 Dahir no. 1-94-124 v. 25.2.1994 portant loi organique no. 29-93 relative au Conseil constitutionnel, Bulletin Officiel no. 4244 v. 2.3.1994.

62 Im Gegensatz zu Art. 56 I frz. Verf., wo eine zweite Amtszeit ausdrücklich ausgeschlossen ist, was eine erhebliche Stärkung der richterlichen Unabhängigkeit bedeutet.

Wie heute noch in Frankreich (Art. $61 \mathrm{ff}$. frVerf). 
Ministerpräsidenten ernennt (Art. 24 n.F.). Das entspricht zwar der bereits geübten Praxis, wurde jetzt aber erst verfassungsmäßig verankert. Der König kann jedoch die Ernennung von Ministern verweigern, wie er selbst geäußert hat, oder mehrere Vorschläge für die Besetzung eines Postens von seinem Premierminister verlangen ${ }^{64}$. Schon deswegen ist die Änderung nicht zu überschätzen. Dazu kommt, daß sich der Ministerpräsident politisch den Wünschen des Königs im Ernstfall nicht verschließen können wird. Außerdem hat sich der König das direkte Abberufungsrecht der Minister vorbehalten. Das ist umso auffälliger, als zur Zeit, als die Verfassungsdiskussion im Gange war, zunächst verlautbart worden war, daß auch das Abberufungsrecht an einen Vorschlag des Premierministers gebunden werden sollte und diese Überlegungen aus nicht erklärten Gründen fallen gelassen wurden $^{65}$.

Weitere Änderungen beziehen sich auf das Verhältnis zwischen Parlament und Regierung. Die Regierung hatte gegenüber dem Parlament eine starke Stellung ${ }^{66}$, und die Möglichkeiten des Parlaments, die Regierung zu kontrollieren, waren bisher recht gering, sie wurden nunmehr an verschiedenen Stellen gestärkt. Eine Regierung muß jetzt bei Amtsantritt dem Parlament ihr Programm vorlegen und ein Vertrauensvotum des Parlaments einholen (Art. 59 III). Ohne dieses kann sie ihre Arbeit nicht aufnehmen. De facto wird durch diese Regelung auch sichergestellt, daß der Ministerpräsident, der ja dem Gesetz nach nur vom Vertrauen des Königs getragen wird, eine Mehrheit im Parlament finden, also der oder einer der größten Parteien angehören muß. Dennoch muß auch diese Regelung vorsichtig interpretiert werden. Durch den Verweis auf Art. 74 II, III ergibt sich wiederum, daß die Mehrheit der Mitglieder des Parlaments der Regierung das Vertrauen verweigern muß, erneut also diese Konstellation, die schwer zu erreichen ist.

Eines der Kontrollinstrumente eines Parlaments gegenüber der Regierung stellt das Fragerecht dar. Auch das marokkanische Parlament hatte zwar von jeher ein Fragerecht gegenüber der Regierung (Art. 55 III), und es wurden auch zahlreiche mündliche und schriftliche Anfragen an die Regierung gerichtet ${ }^{67}$.- Zwar sah die Geschäftsordnung 20 Tage zur Beantwortung für mündliche (Art. 107) und 15 Tage für schriftliche Anfragen (Art. 110) vor.- Dennoch blieben diese jedoch häufig einfach unbeantwortet oder wurden nur verspätet beantwortet ${ }^{68}$. Damit konnte aber, worauf Naciri hinweist, der politische Zweck

67 I. Alaoui, Les questions adressées au Gouvernement, in: Trente années de vie constitutionnelle au Maroc, D. Basri/M. Rousset/G. Vedel (Hrsg.), Paris 1993, 515 ff. (517); in der Zeit von 1984-1992 insgesamt 4039, davon 2344 mündliche und 1695 schriftliche Anf ragen.

68

Laut Alaoui, a.a.O., lag die Zahl der beantworteten Anfragen in der (verlängerten) Legislaturperiode von 1984-1992 bei 82\%. 1963 von 2344 mündlichen und 1351 von 1695 mündlichen Anfragen. Bisweilen wurden Anfragen auch erst nach 1-2 Jahren beantwortet. Dabei gab es auch erhebliche 
einer Anfrage häufig nicht mehr erreicht werden, denn oft kommt es hierfür auf die aktuelle Situation an ${ }^{69}$. Um die "Beantwortungsmoral" zu verbessern, wurde jetzt die Pflicht der Regierung zur Beantwortung von Anfragen mit einer Frist von 20 Tagen in Verfassungsrang erhoben (Art. 55 IV) Was allerdings zu geschehen hat, wenn die Frist trotzdem überschritten wird, bleibt wiederum offen.

Die Verfassung von 1972 sah keine Einsetzung von Untersuchungs- oder Kontrollausschüssen vor. Dennoch wurden in die Parlamentarische Geschäftsordnung vom 20.5.1985 in Art. 44 Untersuchungsausschüsse aufgenommen. Diese wurden aber von der chambre constitutionnelle, die die Parlamentarische Geschäftsordnung routinemäßig auf ihre Verfassungsmäßigkeit zu überprüfen hatte, für verfassungswidrig erklärt, da in dieser nicht vorgesehen. ${ }^{70}$ Nunmehr ermöglicht Art. 40 II-IV die Einrichtung von Untersuchungsausschüssen, allerdings nur auf Antrag der Mehrheit des Parlaments oder des Königs. Als Instrument der Opposition sind sie daher nur begrenzt tauglich.

Als Stärkung des Parlaments ist auch die neue Bestimmung gemeint, daß die Ausrufung des Notstands nicht die Auflösung des Parlaments nach sich zieht (Art. 35 III), während dieses in einem solchen Fall zumindest als suspendiert galt. Hier sollte sehr genau auf den Unterschied in der Formulierung in der französischen Verfassung geachtet werden. Art. $16 \mathrm{~V}$ frz. Verfassung sagt, daß im Notstandsfall das Parlament nicht aufgelöst werden kann ("ne peut etre dissoute"), während der Wortlaut der marokkanischen Regelung nur feststellt, daß die Auflösung des Parlaments nicht die Folge der Erklärung des Notstands ist. Es ist zu beachten, daß der König berechtigt ist, im Notstandsfalle alle notwendigen Maßnahmen zu treffen. Die marokkanische Formulierung enthält kein Verbot einer Parlamentsauflösung, wenn der König sie als Notstandsmaßnahme für geboten hält ${ }^{71}$. Was das Parlament im Ausnahmezustand an Befugnissen behält, wird in der Verfassung nicht deutlich, grundsätzlich ist wohl davon auszugehen, daß es seine Funktionen weiterhin

Unterschiede zwischen den Ministerien und besonders das Ministerium für Inneres und Information und das Justizministerium zeigten sich bei der Beantwortung von Fragen als nachlässig und zogen dadurch besonderen Tadel auf sich, weil sie die Ministerien seien, die bei der Durchsetzung rechtsstaatlicher Verhältnisse in besonderem Maße Verantwortung trügen.

Naciri, 1985, 124.

70 Arrêt de la chambre constitutionnelle no. 182 du 22 août 1985 (Bulletin Officiel no. 3807 du 16 octobre 1985). Allerdings hatte das nicht gehindert, daß tatsächlich je einmal in der Legislaturperiode von 1977-83 und 1984-92 Geschehnisse auf königliche Anordnung hin von einer Art Untersuchungskommission geprüft wurden, $M$. Essaid, Le rôle des commissions parlementaires et du bureau de la Chambre des Représentants, in: Trente années de vie constitutionnelle au Maroc, D. Basri/M. Rousset/G. Vedel (Hrsg.), Paris 1993, 487 ff. (512).

71 Robert, L'état d'exception dans la Constitution, in: Trente années de vie constitutionnelle, D. Basri/M. Rousset/G. Vedel (Hrsg.), Paris 1993, 239 ff. (259) interpretiert die Regelung als Auflösungsverbot, zweif elnd dagegen Menouni, Qira'a fi masru dustur al-muraga (Nachdenkliches Lesen des Entwurfs der revidierten Verfassung), in: Muraga at ad-dustur al-magribi 1992 (Die Revision der marokkanischen Verfassung 1992), D. Basri/M. Rousset/G. Vedel (Hrsg.), o.O. (Rabat) 1992, 106-121 (116). 
wahrnehmen kann, sofern der König diese nicht ausdrücklich durch Notstandsmaßnahmen einschränkt

Von den weiteren Änderungen seien nur noch zwei genannt ${ }^{72}$, einmal die Schaffung von Regionen als Gebietskörperschaften im Verwaltungsaufbau (Art. 94) über Präfekturen, Provinzen und Gemeinden sowie des Rats für Wirtschaftliche und Soziale Fragen (Conseil Economique et Social, Art. 91 ff.), der an die Stelle des Hohen Rats für nationale Förderung und Planung (Conseil Supérieur de la promotion Nationale et du Plan) getreten ist. Auch er ist ein Beratungsorgan, das von einem französischen Vorbild angeregt wurde, aber viel schwächer als dieses ist ${ }^{73}$. Beide Gremien können zwar von Regierung und Parlament um Stellungnahmen zu allen sozialen und wirtschaftlichen Fragen gebeten werden. Während aber der französische Rat bei allen Gesetzgebungsvorhaben auf diesen Gebieten gehört werden muß, fehlt eine solche Pflicht bei dem marokkanischen Rat.

Eine Reihe von Anliegen insbesondere der Oppositionsparteien kamen dagegen bei dieser Verfassungsänderung nicht zum Zuge. Dazu gehörte z.B. eine Änderung des Wahlsystems. Die Oppositionsparteien forderten, daß alle Abgeordneten durch allgemeine direkte Wahlen gewählt werden sollten, weil das bisherige Wahlverfahren, nach dem ein Drittel der Abgeordneten zu einem jeweils späteren Termin als dem Wahltermin für die ersten beiden Drittel in indirekter Wahl gewählt wurde, zu leicht die Möglichkeit bot, Wahlen in erwünschte Richtungen zu beeinflussen ${ }^{74}$. Auch eine Verkürzung der Legislaturperiode von 6 auf 5 Jahre war nicht durchsetzbar. Gleiches gilt für das Bestreben, der Regierung nach dem Vorbild der französischen Verfassung (Art. 20 frz. Verf.) die Formulierung der allgemeinen Politik des Staates zu übertragen, die Gesetzgebungszuständigkeiten des Parlaments auszuweiten, z.B. auf Gnadenrecht, Wahlrecht und die Ratifikation von internationalen Verträgen. Die Einsetzung eines Verteidigungsrats, eines Informationsrats, eines Erziehungs- und Bildungsrats sowie eines Rechnungshofs gehört ebenfalls zu den Anliegen, die noch nicht verwirklicht-werden konnten ${ }^{75}$. 


\title{
Constitutional Development in Morocco
}

\author{
By Silvia Teilenbach
}

In 1992 the Moroccan Constitution was considerably revised with a view to strengthening the observance of human rights and the rule of law. The most three important groups of amendments concerned the preamble, in which the attachment of Morocco to human rights as they are universally recognized, is affirmed, the relationship of parliament and government, which has been remoulded to strengthen the position of parliament, and the introduction of a constitutional council, which has much larger competences of constitutional control than the former constitutional senate of the Supreme Court. The article describes the changes in detail and tries to explain them in light of their political background.

\section{The Juridical and Political Functions of Social Norms (The Case of Sub-Saharan Africa)}

\section{By Paul Trappe}

Since centuries it has been realized, that the legal norms rest on a basis of social norms. Both organize the social order as a whole. We find hints on this phenomenon of a dual organization above all in the writings of the French sociologists E. Durkheim, G. Gurvitch, and J. Carbonnier. A. de Tocqueville can be regarded as a forerunner with his 'American Democracy'.

Legal and social spheres are intermingling with each other in complex social systems as well as in systems which appear less complex, as in Africa South of the Sahara (ASS) where strong segmentary roots of social organization still exist. Great parts of social interrelation go along the lines of traditional social norms of ethnic groups, kin groups, family and neighbourhood units, "agrégats" (E. Durkheim) of autochthonous segmentary systems.

ASS has undergone a shift in social organization from kin groups to functional groups, in which non-relative members join for clear cut aims. Group pluralism is growing up, but have not yet come to a degree similar to that in industrialized nation States of the West. In all societies of the world there is a trend towards more self-organizing pluralism (N. Elias). These trends and facts have consequences for the adoption of legal norms.

The most striking ones are to be seen in the necessity to develop institutions beyond the given social systems, especially on the intermediate level. The group norms must be regarded as a complementary order for the law of the nation State. 